\title{
DEFORMABLE OBJECT TRACKING USING OPTICAL FLOW CONSTRAINTS
}

\author{
A. Hilsmann , P. Eisert \\ Fraunhofer Institute for Telecommunications, Heinrich-Hertz Institute \\ Einsteinufer 37, D-10587 Berlin, Germany \\ \{anna.hilsmann, peter.eisert\}@ @hi.fraunhofer.de
}

Keywords: deformable object tracking, optical flow constraint, deformable meshes

\begin{abstract}
In this paper, we present a method for deformable object tracking that exploits the entire image information using the optical flow equation instead of working with discrete feature points. Our method starts from the optical flow constraint and first estimates global transformations in a hierarchical framework. Elastic deformations are then estimated separately using deformable meshes and spatial and temporal smoothing constraints. In both cases additional constraints to regularize the optical flow field are obtained from the motion model such that finding the best transformation amounts in minimizing an error function that can be solved in a least squares sense.

Combing deformable meshes and the optical flow equation with a dedicated weighted smoothness constraint on the mesh deformation and estimating global transformations separately from elastic deformations is key to dealing with complex deformations such as cloth deformation as a person moves.
\end{abstract}

\section{Introduction}

Deformable object tracking has many fields of application including medical imaging [16], object-based video compression [7], [17] or augmented reality [19]. It has been successfully demonstrated for human motion analysis and face tracking. We are particular interested in images of surfaces whose deformations are difficult to describe, such as drapery of textiles. Automated tracking of deformations in $2 \mathrm{D}$ images is challenging because the complexity of the deformation field is unknown a priori and therefore the assumed deformation model has to cope with various types of deformations.

One solution to this problem is to compute a regularized optical flow field. As the optical flow equation provides one equation for two unknowns additional constraints are needed. These can be smoothing constraints on the optical flow field itself. We provide them by explicit motion models.

The main contribution of this paper is the introduction of deformable $2 \mathrm{D}$ triangle meshes along with an error function based on the optical flow equation that determines the mesh deformation in each frame, i.e. the displacement of each vertex. Each pixel position of the image can be represented as a weighted sum of surrounding mesh vertices. Fitting then amounts in minimizing an error function that consists of two parts. The first part formulates the error given by the optical flow constraint for a particular deformation model. The second part is a formulation of mesh smoothness in the neighborhood of each vertex. Global transformations and elastic deformations are estimated separately which allows us to regularize the vertex displacements in the deformation estimation steps without inhibiting smooth global transformations.

The application we have in mind is a real-time application in a virtual mirror environment such as the one presented in [9]. In this application a person steps in front of the virtual mirror and instead of seeing oneself in a real mirror, deformable objects like a logo on a t-shirt can be tracked and e.g. changed in color or be replaced by another logo to enable virtual dressing (see Figure 1).

The remainder of this paper is structured as follows. We first briefly review the existing literature in Section 2 before we present our algorithm in Section 3. In Section 4 we present the results we obtained with our algorithm.

\section{Related work}

Motion between two frames is commonly described by a dense displacement vector field which links the location of each point in a given frame to its location in the next frame. Registering a model to an image and tracking of deformable objects is a large field of research and there exists a multitude of methods. Basically the literature distinguishes between intensity- and feature-based methods. Feature-based methods minimize a distance between a few corresponding features whereas direct methods minimize an error measure that is based on image information at all pixels in the image.

Feature-based methods determine correspondences between distinct features and use them to estimate the best transformation between these correspondences. Various features have been described in the literature. Image-based feature points are e.g. local curvature extrema or saddle points, edges or corners [5], [20], [11] or SIFT features [15]. Popular choices of shape-features are the shape context approach of Belongie et al. [2], or statistical moments of the shape [4]. Feature- based methods are mostly used to find 
rigid or affine transformation. In order to obtain a smooth motion field from the discrete feature point correspondences an explicit motion model like an affine transform or the 12parameter model can be fitted to the points by minimizing some error function. If the set of points is large enough also more complex transformations can be determined using e.g. radial basis functions (RBF) [3], [10]. The type of the RBF determines overall characteristics of the transformation such as the smoothness or the locality.

In [18] a feature-based real-time method for deformable object detection and tracking is presented using a widebaseline matching algorithm [14] and deformable meshes. Our approach is similar to theirs referring to the motion model but we use direct image information instead of distinct keypoints. Additionally, they do not separate global motion from elastic deformations such that regularizing the displacement vectors of the mesh vertices inhibits large but smooth global transformations.

Intensity-based methods do not rely on features and fit the model directly to the image data [13] mainly using the optical flow equation [12]. The optical flow constraint is ill-posed as it provides one equation for two unknowns. Therefore additional constraints are required. These can be provided by additional smoothing constraints or by a predefined motion model to regularize the optical flow field. They have been successfully demonstrated in the context of face tracking [6], [8] using deformable meshes or for camera motion or person tracking using rigid or affine motion models.

In [1] a method has been presented that uses optical flow in connection with radial basis functions to track less constrained deformations. Their method uses an iterative scheme to adapt the number of RBF centers to the degree of non-rigidity between the images.

The next section describes our optical flow based tracking approach using deformable meshes.

\section{Optical Flow based deformable object tracking}

In order to track an object in a video sequence we make use of the optical flow constraint equation [12]:

$$
I_{x}\left(x_{i}, y_{i}\right) \cdot d_{x}\left(x_{i}, y_{i}\right)+I_{y}\left(x_{i}, y_{i}\right) \cdot d_{y}\left(x_{i}, y_{i}\right)=-I_{t}\left(x_{i}, y_{i}\right)
$$

where $I_{x}\left(x_{i}, y_{i}\right)$ and $I_{y}\left(x_{i}, y_{i}\right)$ are the spatial derivatives of the image at pixel position $\left[x_{i}, y_{i}\right]^{T}$ and $I_{t}\left(x_{i}, y_{i}\right)$ denotes the intensity change between two images, in our case between our deformed model and the current frame of the video sequence. In order to be independent from lighting changes, we first segment the objects of interest and work with greyscale images with pixel values 0 in the background and higher values for the foreground object. Hereby we filter both images with a moving average filter transforming the binary object borders into linear ramps. $d\left(x_{i}, y_{i}\right)$ denotes the displacement vector at position $\left[x_{i}, y_{i}\right]^{T}$ that transforms the pixel $\left[x_{i}, y_{i}\right]^{T}$ from the previous

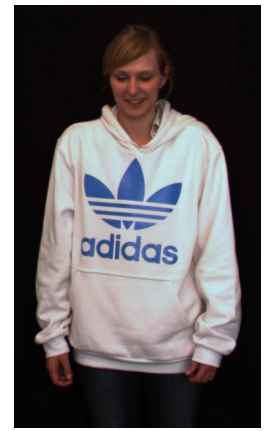

(a) Captured scene

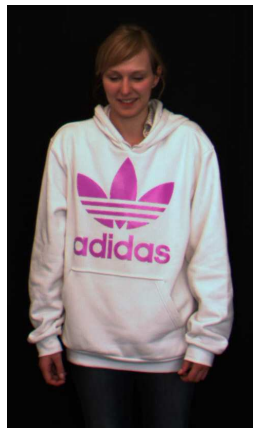

(b) Color of the logo changed

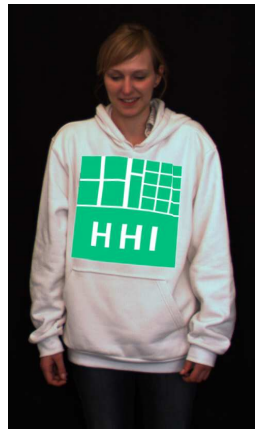

(c) Logo replaced by another $\log 0$

\section{Figure 1: Possible application of the approach}

frame onto the pixel $\left[x_{i}^{\prime}, y_{i}^{\prime}\right]^{T}$ in the current frame:

$$
d_{i}=\left[x_{i}^{\prime}, y_{i}^{\prime}\right]^{T}-\left[x_{i}, y_{i}\right]^{T}
$$

However, the solution to (1) is under-determined as each equation has two unknowns. Additional constraints can be provided by smoothing constraints on the optical flow field or by a predefined motion model, e.g. a global motion model as described in section 3.1 or a deformation model as depicted in section 3.2. Finding the best transformation then amounts into minimizing the quadratic error

$$
\begin{aligned}
E=\sum_{i=1}^{n} & \| I_{x}\left(x_{i}, y_{i}\right) \cdot d_{x}\left(x_{i}, y_{i}\right) \\
& \quad+I_{y}\left(x_{i}, y_{i}\right) \cdot d_{y}\left(x_{i}, y_{i}\right)+I_{t}\left(x_{i}, y_{i}\right) \|_{2}
\end{aligned}
$$

with $n$ being the number of pixels selected for contribution to the error function, i.e. pixels where the gradient is non-zero.

\subsection{Estimating global transformations in a hierarchical framework}

The optical flow constraint (1) is valid only for small displacements because it assumes the image intensity to be linear between two successive frames. In order to cope with larger displacements a hierarchical framework can be used [8]. For our purpose we use an image pyramid with three levels, but more levels are easily applicable. On the lowest level where the linear intensity assumption is valid in a wider range we estimate a global translation of the whole object, i.e. the displacement vectors are the same for every pixel position

$$
\begin{gathered}
d_{x}\left(x_{i}, y_{i}\right)=d_{x} \\
d_{y}\left(x_{i}, y_{i}\right)=d_{y} .
\end{gathered}
$$

The best translation is then found by inserting the global translation model given by equation (4) into equation (3). Minimizing the error results in an equation system with $n$ equations and 2 unknowns which is solved in a least squares sense.

The translation estimated on the first level is applied on the second level and a warped image is computed. From this image 


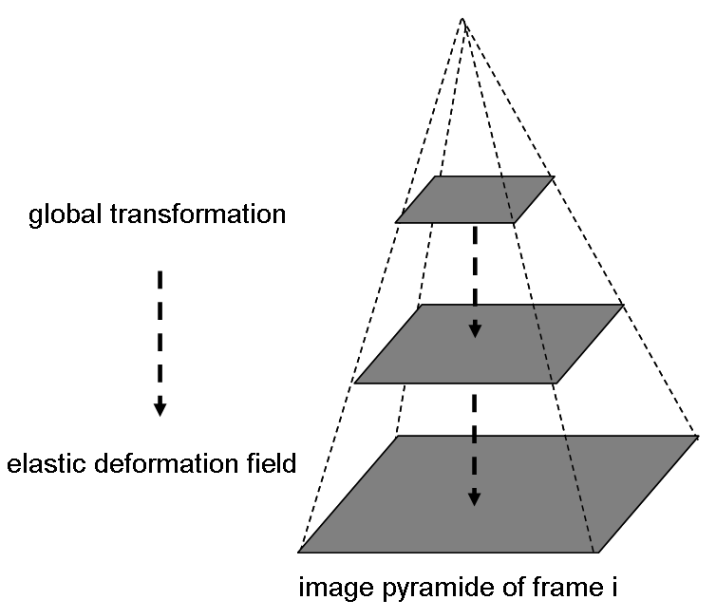

Figure 2: Hierarchical framework

we estimate an affine transformation of the object on the second level. If $\mathbf{A}$ is the affine matrix that maps $\left[x_{i}, y_{i}\right]^{T}$ on $\left[x_{i}^{\prime}, y_{i}^{\prime}\right]^{T}$ with

$$
\begin{array}{r}
{\left[x_{i}^{\prime}, y_{i}^{\prime}, 1\right]^{T}=\mathbf{A} \cdot\left[x_{i}, y_{i}, 1\right]^{T}} \\
\mathbf{A}=\left(\begin{array}{ccc}
a_{1} & a_{2} & a_{3} \\
a_{4} & a_{5} & a_{6} \\
0 & 0 & 1
\end{array}\right)
\end{array}
$$

the displacement vector $d\left(x_{i}, y_{i}\right)$ can be written as

$$
\begin{gathered}
d_{x}\left(x_{i}, y_{i}\right)=\left(a_{1}-1\right) \cdot x_{i}+a_{2} \cdot y_{i}+a_{3} \\
d_{y}\left(x_{i}, y_{i}\right)=a_{4} \cdot x_{i}+\left(a_{5}-1\right) \cdot y_{i}+a_{6} .
\end{gathered}
$$

The best affine transformation is then found by inserting the affine motion model given by equation (6) into equation (3). Minimizing the error results in an equation system with $n$ equations and 6 unknowns which is solved in a least squares sense.

We combine the translation and affine transformation estimated on the first two levels and apply the resulting transformation on the next level. We compute a warped image and calculate the remaining elastic deformations of the object using a triangulated mesh as described in the next section. The hierarchical approach is depicted in Figure 2.

The estimation of global transformations in a coarse-to-fine approach serves a dual purpose. First, we can cope with larger global transformations. Second, estimating global transformations separately from the deformations on the last level allows us to put smoothing constraints on the vertex displacements without inhibiting large but smooth global transformations of the whole shape.

\subsection{Optical flow based elastic deformation estimation}

We present our model $\mathrm{M}$ as a triangulated regular 2D mesh with $m$ vertices $\mathbf{v}_{\mathbf{k}},(k=1 \ldots m)$ such as shown in Figure 3. The position of each vertex $\mathbf{v}_{\mathbf{k}}$ is given by its image coordinates $\mathbf{p}_{\mathbf{i}}=\left[x_{k}, y_{k}\right]^{T}$. Each pixel $\mathbf{p}_{\mathbf{i}}=\left[x_{i}, y_{i}\right]^{T}$ in the image can be represented by its barycentric coordinates of its enclosing triangle $t$ :

$$
\begin{aligned}
{\left[x_{i}, y_{i}\right]^{T}=} & \sum_{\substack{j=1 \\
\text { jit } \in \mathbf{v}_{\mathbf{k}}}}^{3} B_{j t}\left(x_{i}, y_{i}\right) \cdot\left[v_{j t x}, v_{j t y}\right]^{T} \\
& \sum_{j=1}^{3} B_{j t}\left(x_{i}, y_{i}\right)=1,0 \leq B_{j t} \leq 1
\end{aligned}
$$

where $B_{j t}\left(x_{i}, y_{i}\right),(j=1,2,3)$ are the three barycentric coordinates of pixel $\left[x_{i}, y_{i}\right]^{T}$ computed on the original mesh and $\mathbf{v}_{\mathbf{j t}}$ are the three vertices of the enclosing triangle $t$ of the mesh consisting of vertices $\mathbf{v}_{\mathbf{k}}$.

With a mesh deformation, $\left[x_{i}, y_{i}\right]$ is mapped onto $\left[x_{i}^{\prime}, y_{i}^{\prime}\right]$. Thus, we are looking for a deformation of the mesh, i.e a displacement of each vertex $\mathbf{v}_{\mathbf{k}}$ to $\mathbf{v}_{\mathbf{k}}^{\prime}$ such that the barycentric coordinates of $\left[x_{i}^{\prime}, y_{i}^{\prime}\right]^{T}$ are those of $\left[x_{i}, y_{i}\right]^{T}$ :

$$
\begin{aligned}
\mathbf{d}_{\mathbf{i}} & =\left[x_{i}^{\prime}, y_{i}^{\prime}\right] \\
& =\sum_{\substack{j=1 \\
\mathbf{v}_{\mathbf{j}}^{\prime} \in \mathbf{v}_{\mathbf{k}}^{\prime}}}^{3} B_{j}\left(x_{i}, y_{i}\right) \cdot\left[v_{j x}^{\prime}, v_{j y}^{\prime}\right]^{T} \\
& -\sum_{\substack{j=1 \\
\mathbf{v} \in \mathbf{j}}}^{3} B_{j}\left(x_{i}, y_{i}\right) \cdot\left[v_{j x}, v_{j y}\right]^{T} \\
& =\sum_{\substack{j=1 \\
\mathbf{d}_{\mathbf{j}} \in \mathbf{d}_{\mathbf{k}}}}^{3} B_{j}\left(x_{i}, y_{i}\right) \cdot\left[d_{j x}, d_{j y}\right]^{T}
\end{aligned}
$$

where $\mathbf{d}_{\mathbf{k}}=\mathbf{v}_{\mathbf{k}}^{\prime}-\mathbf{v}_{\mathbf{k}}$ denotes the displacements of the mesh vertices. Thus, the deformation model can be stated as:

$$
\begin{aligned}
& d_{x}\left(x_{i}, y_{i}\right)=\sum_{\substack{j=1 \\
t_{j} \in t_{k}}}^{3} B_{j}\left(x_{i}, y_{i}\right) \cdot d_{j x} \\
& d_{y}\left(x_{i}, y_{i}\right)=\sum_{\substack{j=1 \\
t_{j} \in t_{k}}}^{3} B_{j}\left(x_{i}, y_{i}\right) \cdot d_{j y} .
\end{aligned}
$$

This leads to an overdetermined linear equation system with $n$ equations and $2 m$ unknowns that is solved in a least-squares sense minimizing the quadratic error given by equation (3).

In order to make the mesh deformation temporally smooth we apply a regularization method minimizing

$$
\begin{aligned}
E=\sum_{i=1}^{n} & \| I_{x}\left(x_{i}, y_{i}\right) \cdot d_{x}\left(x_{i}, y_{i}\right) \\
& +I_{y}\left(x_{i}, y_{i}\right) \cdot d_{y}\left(x_{i}, y_{i}\right)+I_{t}\left(x_{i}, y_{i}\right) \|_{2} \\
& +\lambda \sum_{k=1}^{m}\left\|\mathbf{d}_{\mathbf{k}}\right\|_{2}
\end{aligned}
$$

where $\lambda$ is the regularization parameter and $\mathbf{d}_{\mathbf{k}}$ are the displacements of the mesh vertices $\mathbf{v}_{\mathbf{k}}$. As we have estimated 
the global transformation in a previous step the displacement vectors of each vertex represent real deformations and no smooth transformation of the whole object. Therefore, the regularization part serves the purpose of smoothing the deformation temporally forcing the displacement to be as small as possible and does not inhibit large but smooth transformations of the shape.

The displacement of each vertex is calculated only from image gradients in the surrounding triangles. Therefore, some vertices are less constrained than others. For example, the displacement of the vertex in the right upper corner of the mesh in Figure 3(b) is only determined by a few points lying close to the triangle edge. Moreover, they are likely to move out of this triangle due to small registration inaccuracies. Thus, this vertex is badly or not at all constrained except for the temporal regularization forcing the displacement to be as small as possible from frame to frame. This problem is depicted schematically in Figure 4. Figure 4(a) shows a mesh containing object boundary points. The triangle consisting of vertices $\mathbf{v}_{\mathbf{0}}, \mathbf{v}_{\mathbf{2}}$ and $\mathbf{v}_{\mathbf{3}}$ does not contain any object boundary points. Thus, the displacement $\mathbf{d}_{\mathbf{3}}$ of vertex $\mathbf{v}_{\mathbf{3}}$ is not constrained by the optical flow equation (1). The regularization term forces the vertex displacements to be as small as possible which can be interpreted as temporal smoothness. Therefore a displacement is zero if it is not constrained by any other equation. So, even if all surrounding vertices have the same displacement vector the displacement of $\mathbf{v}_{\mathbf{3}}$ is zero resulting in a deformation of the triangle (see Figure 4(b)). In order to obtain a spatially smooth result we want the vertex to behave like its neighbors (see Figure 4(c)) and need additional constraints. In order to restrict the displacements of such under-constrained vertices and to make the mesh deformation spatially smooth we incorporate additional equations such that now the error

$$
\begin{aligned}
E=\sum_{i=1}^{n} & \| I_{x}\left(x_{i}, y_{i}\right) \cdot d_{x}\left(x_{i}, y_{i}\right) \\
& +I_{y}\left(x_{i}, y_{i}\right) \cdot d_{y}\left(x_{i}, y_{i}\right)+I_{t}\left(x_{i}, y_{i}\right) \|_{2} \\
& +\lambda \sum_{k=1}^{m}\left\|\mathbf{d}_{\mathbf{k}}\right\|_{2} \\
& +\sum_{k=1}^{m} w_{k} E_{s}\left(\mathbf{d}_{\mathbf{k}}\right)
\end{aligned}
$$

is minimized, where $E_{s}\left(\mathbf{d}_{\mathbf{k}}\right)$ is some smoothing function for the vertex displacement $\mathbf{d}_{\mathbf{k}}$ weighted by $w_{k}$. We chose $E_{s}\left(\mathbf{d}_{\mathbf{k}}\right)$ to be

$$
\begin{aligned}
E_{s}\left(d_{k}\right) & =\left\|d_{k x}-\frac{1}{\left|N_{k}\right|} \sum_{n \in N_{k}} d_{n x}\right\|_{2} \\
& +\left\|d_{k y}-\frac{1}{\left|N_{k}\right|} \sum_{n \in N_{k}} d_{n y}\right\|_{2}
\end{aligned}
$$

where $N_{k}$ denotes the set of neighbor vertices of vertex $\mathbf{v}_{\mathbf{k}}$, i.e. all vertices $\mathbf{v}_{\mathbf{k}}$ is connected to. $E\left(\mathbf{d}_{\mathbf{k}}\right)$ is a measure of vertex' displacement deviation to the displacements of its

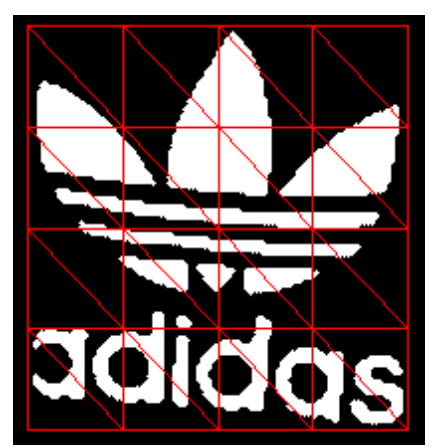

(a) undeformed mesh on the binary model frame

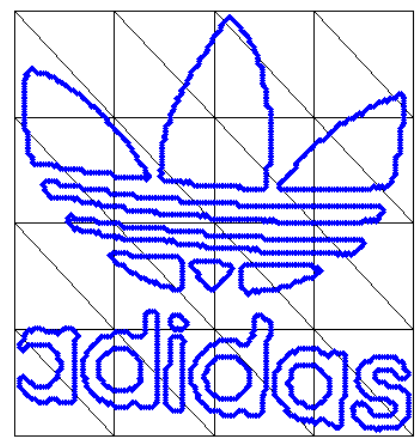

(b) undeformed mesh on boundary sample points

\section{Figure 3: Undeformed mesh on the model frame}

neighbors. In the example presented in Figure 4 where the neighbor displacements are equal $\left(\mathbf{d}_{\mathbf{0}}=\mathbf{d}_{\mathbf{2}}\right)$ this error is minimized for $\mathbf{d}_{\mathbf{3}}=\mathbf{d}_{\mathbf{0}}=\mathbf{d}_{\mathbf{2}}$ which results in an undistorted triangle.

The weight $w_{k}$ can be e.g. adapted to the degree of 'constrainedness' of a vertex $\mathbf{v}_{\mathbf{k}}$, e.g. the number of image points that influence its displacement. The less pixels contribute to the vertex displacement the higher the weight $w_{k}$ to the additional smoothness constraint and the more the vertex displacement is forced to equal the surrounding displacements. We chose $w_{k}$ to be reciprocal to the norm of all linear factors of $d_{k x}$ and $d_{k y}$ in the linear equation system we obtain from equation (3) and (7)

$$
w_{k} \propto K \cdot \frac{1}{\left\|\alpha_{\mathbf{k x}}\right\|_{2}+\left\|\alpha_{\mathbf{k y}}\right\|_{2}}
$$

where $\alpha_{\mathbf{k x}}$ and $\alpha_{\mathbf{k y}}$ are the vectors of linear factors of $d_{k x}$ and $d_{k y}$ in the linear equation system. The factor $K$ controls the trade- off between mesh-smoothness and mapping accuracy.

\subsection{D tracking}

For our purpose we use the first segmented video frame as model represented by the triangulated mesh $M_{1}=$ $M\left(\mathbf{v}_{\mathbf{k} \mathbf{1}}, \mathbf{e}_{\mathbf{k} \mathbf{1}}\right)$, consisting of vertices $\mathbf{v}_{\mathbf{k} \mathbf{1}}$ and edges $\mathbf{e}_{\mathbf{k} \mathbf{1}}$. We start by estimating a global transformation $T_{1,2}$ from the first frame $I_{1}$ to the next frame $I_{2}$ in a hierarchical framework as described in Section 3.1. The global transformation $T_{1,2}$ is applied to the mesh and a globally warped image is computed from $I_{1}$. From this image we calculate the remaining elastic deformations of the mesh, i.e. vertex displacements $\mathbf{d}_{\mathbf{k} \mathbf{1 , 2}}$, as depicted in Section 3.2. The complete transformation (global transformation and elastic deformation) is now defined by the new mesh $M_{2}=M\left(\mathbf{v}_{\mathbf{k} 2}, \mathbf{e}_{\mathbf{k 2}}\right)$ consisting of new vertex positions:

$$
\mathbf{v}_{\mathbf{k} \mathbf{2}}=\mathbf{v}_{\mathbf{k} \mathbf{1}}^{\prime}=T_{1,2}\left(\mathbf{v}_{\mathbf{k} \mathbf{1}}\right)+\mathbf{d}_{\mathbf{k} \mathbf{1}, \mathbf{2}}
$$

The new mesh $M_{2}$ can now be used to compute a warped frame $I_{2}^{\prime}$ from $I_{1}$. In the next step we estimate a global transformation $T_{2^{\prime}, 3}$ and elastic deformations $\mathbf{d}_{\mathbf{k} \mathbf{2}^{\prime}, 3}$ from the warped image $I_{2}^{\prime}$ 


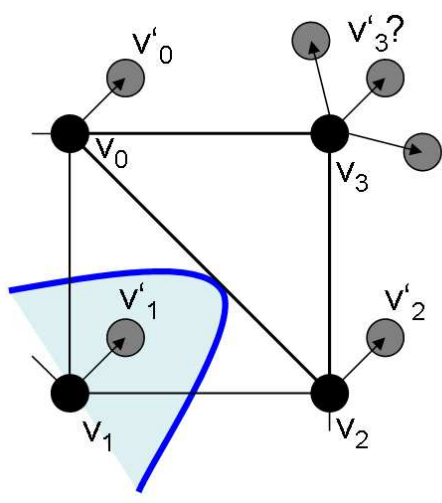

(a)

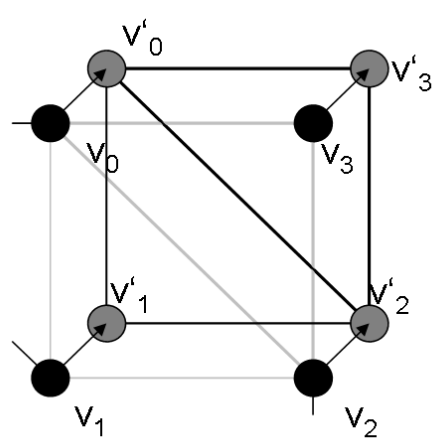

(b)

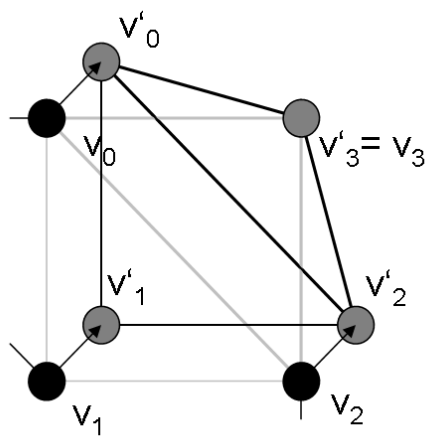

(c)

Figure 4: Illustration of the smoothness constraint for underdetermined vertices

to the third frame $I_{3}$ based on the new mesh $M_{2}$. We can now use the complete deformed mesh $M_{3}$ to warp the first frame $I_{1}$ onto the third frame $I_{3}^{\prime}$.

In each following step we estimate a global transformation $T_{n^{\prime} n+1}$ and elastic deformations $\mathbf{d}_{\mathbf{k n}^{\prime}, \mathbf{n}+\mathbf{1}}$ from the warped image $I_{n}^{\prime}$ to frame $I_{n+1}$, i.e. in each step the mesh is deformed a bit more, and we use the complete mesh deformation to warp the first frame onto frame $I_{n+1}$.

Using the warped images for the estimation of the transformation instead of the frames themselves avoids error accumulation in the transformation. The approach is depicted in Figure 5.

\section{Results}

We applied the method described in the previous section to a video sequence of 160 frames tracking the elastic deformations of a logo on a shirt (see Figure 6) including partial occlusion (e.g. in Figure 6(e)) and 3D rotation (e.g. Figure 6(g),6(h) ).

Figure 7 depicts the mesh deformation using the error function defined in equation (10). To illustrate the mapping result we use the contour of the logo that we find using a simple gradient operator and overlay the contour of the deformed model (in blue in Figure 7) with the contour of the target object (in red in Figure 7).

As denoted above some vertex displacements are more constrained than others, i.e. the vertices belong to different numbers of triangles on the one hand and these triangles contain different numbers of gradient pixels on the other hand. For example, the displacement of the vertex in the right upper corner of the mesh is constrained only by a few contour points of the logo lying close to the triangle edge. Moreover, e.g. in Figure 6(f) the target object has moved out of the triangle which means that the vertex displacement is badly or not at all constrained besides the regularization term forcing the displacement to be as small as possible. However, a better, i.e. smoother result would be obtained if the vertex behaved like the other vertices belonging to the same triangle as stated in Section 3.2, resulting in a minimal distortion of the triangle. Figure 8 depicts the mesh deformation we obtain using the error function given in equation (12). In this case the displacement of the vertex in the right upper corner is constrained by its neighbors which results in a smoother version of the mesh deformation.

From both results we learn that although the estimated mesh deformation cannot represent the partial occlusion in Figure 6(e), 7(e) and 8(e) accurately the system does not lose track because we estimate the transformations from the warped images allowing for recovering from such estimation inaccuracies (see Figures 7 and 8).

\section{Conclusion and outlook}

We presented a method to track deformations of an object in a pre-segmented binary video sequence using the optical flow constraint. We estimate global transformations and elastic deformations separately in a hierarchical framework which allows us to put temporal smoothing constraints on the transformation in the elastic deformation estimation step without inhibiting global large but smooth transformations. Additionally, we introduce spatial smoothness constraint equations on the vertex displacements that we weight according to the 'constrainedness' of a vertex by the optical flow equation. The less a vertex displacement is constrained by the optical flow the higher the weight and the more the displacement is forced to smooth the mesh. By estimating the transformations from warped images instead of the frames themselves we allow for recovering from previous inaccuracies in the transformation estimation.

Next steps will concentrate on extending the method to nonbinary video-sequences, so that the pre-segmentation step is not needed anymore. Moreover, using hierarchical meshes allows us to refine the mesh in regions where the deformation is large. Further effort will be made, to define simple shape features for detection of the desired object in the video frame for initialization. The objective is to design a real-time deformable object tracking system so that further steps will also concentrate on real-time application of the method. 


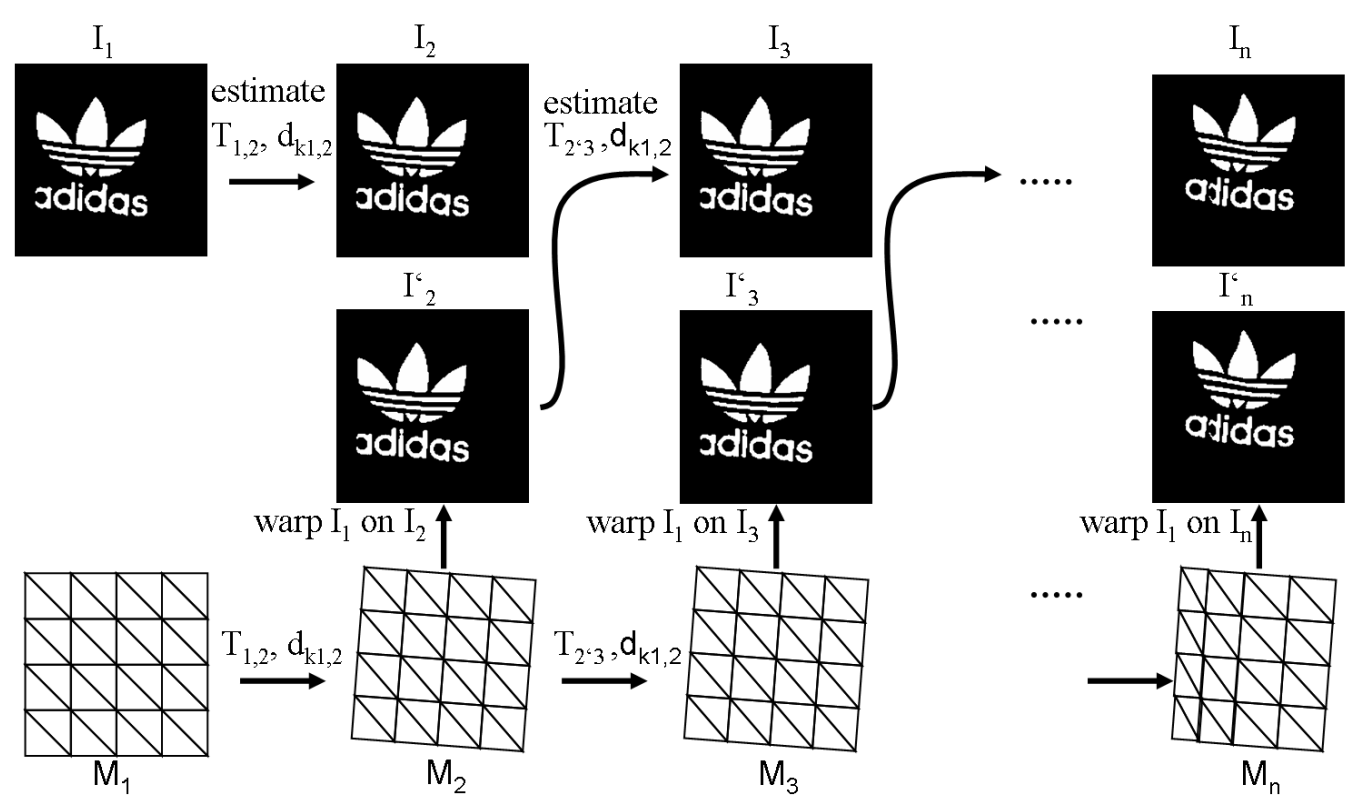

Figure 5: Illustration of the tracking approach

\section{Acknowledgements}

The work presented in this paper has been developed with the support of the European Network of Excellence VISNET II (Contract IST-1-038398).

\section{References}

[1] A. Bartoli and A. Zisserman. Direct estimation of nonrigid registrations. In Proc. 15th British Machine Vision Conference, 2004.

[2] S. Belongie, J. Malik, and J. Puzicha. Shape Matching and Object Recognition Using Shape Contexts. IEEE Transactions on Pattern Analysis and Machine Intelligence, 24(24), pp. 509-510, April 2002.

[3] F. L. Bookstein. Principal Warps: Thin-Plate Splines and the Decomposition of Deformations. IEEE Transactions on Pattern Analysis and Machine Intelligence, 11(6), pp. 567-585, June 1989.

[4] C. C. Chen. Improved Moments Invariants for Shape Discrimination. Pattern Recognition, 26(5), pp. 683-686, 1993.

[5] C. S. Chua and R. Jarvis. 3D Free-Form Surface Registration and Object Recognition. International Journal of Computer Vision, 17, pp. 77-99, 1996.

[6] D. DeCarlo and D. Metaxas. Deformable model-based shape and motion analysis from images using motion residual error. In Proc. 6th International Conference on Computer Vision (ICCV), USA, p.113, 1998.
[7] P. Eisert. Mpeg-4 facial animation in video analysis and synthesis. International Journal of Imaging Systems and Technology, 13(5), March pp. 245-25, 2003.

[8] P. Eisert and B. Girod. Analyzing facial expressions for virtual conferencing. IEEE Comput. Graph. Appl., 18(5), pp. 70-78, 1998.

[9] P. Eisert, J. Rurainsky, and P. Fechteler. Virtual Mirror: Real-Time tracking of Shoes in Augmented Reality Environments. In Proc. International Conference on Image Processing (ICIP'07), 2007.

[10] M. Fornefett, K. Rohr, and H. S. Stiehl. Elastic Registration of Medical Images Using Radial Basis Functions with Compact Support. In Proc. Computer Vision and Pattern Recognition (CVPR'99), pp. 402-407, June 1999.

[11] C. Harris and M. Stephens. A combined corner and edge detection. In Proc. 4th Alvey Vision Conference, pp.147$151,1988$.

[12] B. K. P. Horn. Robot Vision. McGraw-Hill Higher Education, 1986.

[13] M. Irani and P. Anandan. About direct methods. In Proc. International Workshop on Vision Algorithms (ICCV'99), London, UK, pp. 267-277, 2000.

[14] V. Lepetit, P. Lagger, and P. Fua. Randomized trees for real-time keypoint recognition. In Proc. Computer Vision and Pattern Recognition (CVPR'05), Washington,USA, pp.775-781, 2005.

[15] D. Lowe. Distinctive Image Features from ScaleInvariant Keypoints. International Journal of Computer Vision, 60(2), pp. 91-110, November 2003. 


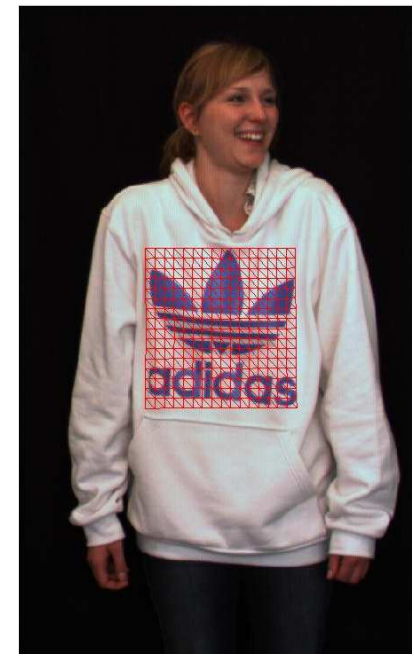

(a)

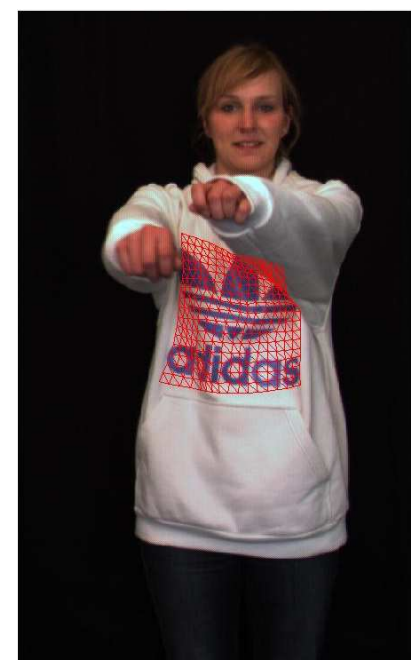

(e)

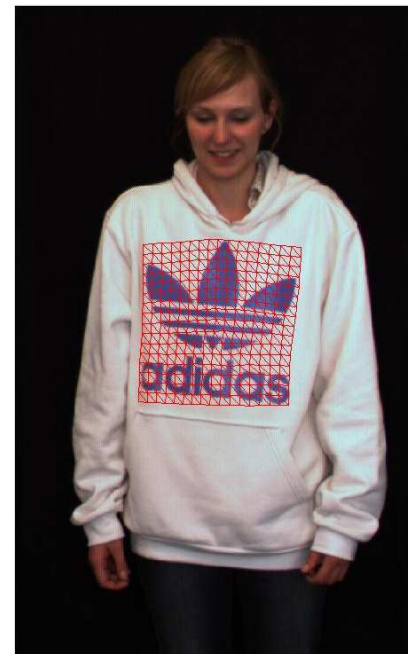

(b)

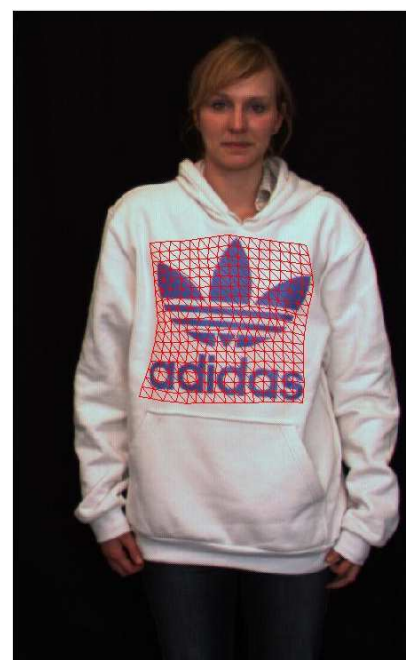

(f)

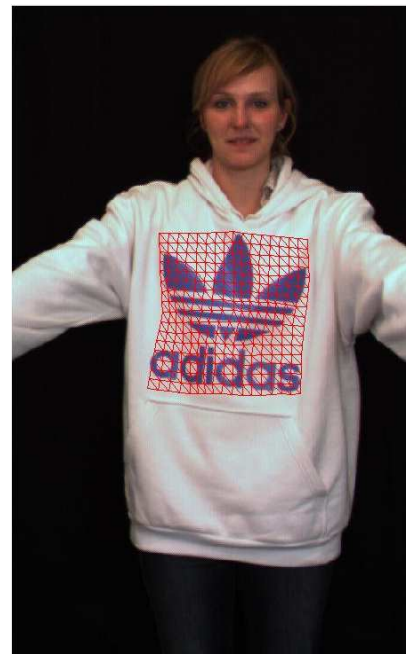

(c)

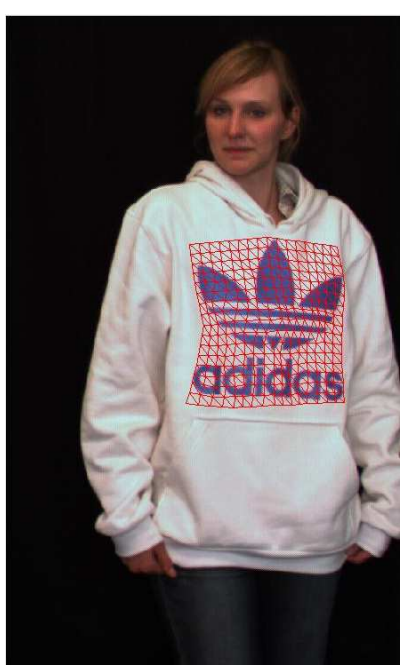

(g)

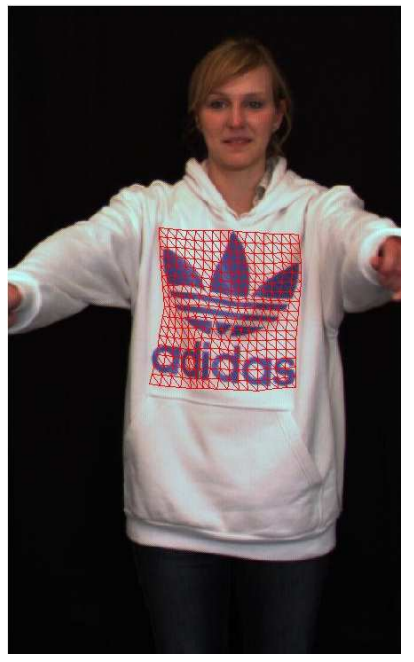

(d)

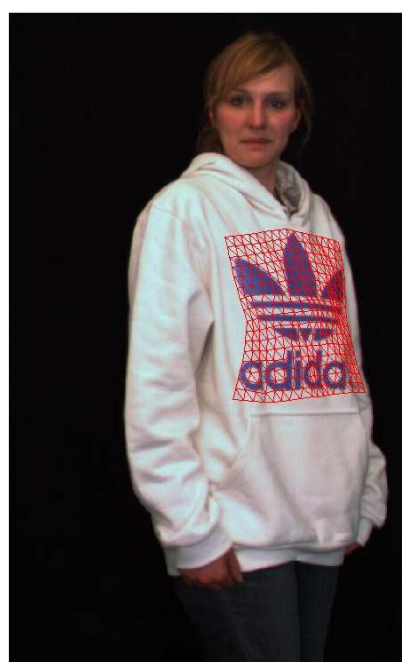

(h)

Figure 6: Example frames of the test sequence with the deformed mesh mapped onto the logo

[16] D. N. Metaxas. Physics-Based Deformable Models: Applications to Computer Vision, Graphics, and Medical Imaging. Kluwer Academic Publishers, Norwell, MA, USA, 1996.

[17] J. Ostermann. Object-oriented analysis-synthesis Coding (OOASC) based on the source model of moving flexible 3D-objects. IEEE Trans. on Image Processing, 3(5), January pp.705-711, 1994.

[18] J. Pilet, V. Lepetit, and P. Fua. Real-time non-rigid surface detection. In Proc Conference on Computer Vision and Pattern Recognition,(CVPR'05), San Diego,USA, 2005.

[19] J. Pilet, V. Lepetit, and P. Fua. Fast non-rigid surface detection, registration and realistic augmentation. International Journal of Computer Vision, January 2007.

[20] J. Thirion. New Feature Points Based on Geometric Invariants for 3D Image Registration. International Journal of Computer Vision, 18(2), pp. 121-137, 1996. 


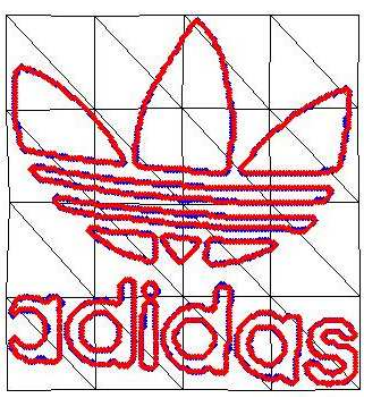

(a)

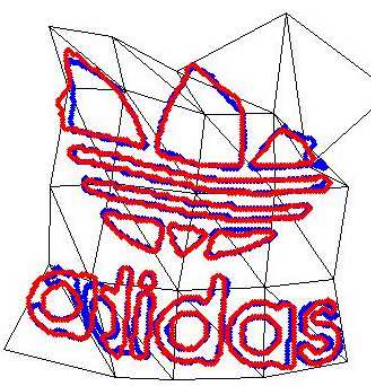

(e)

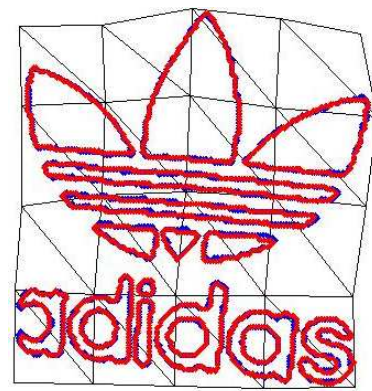

(b)

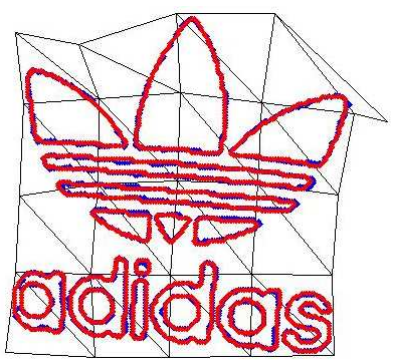

(f)

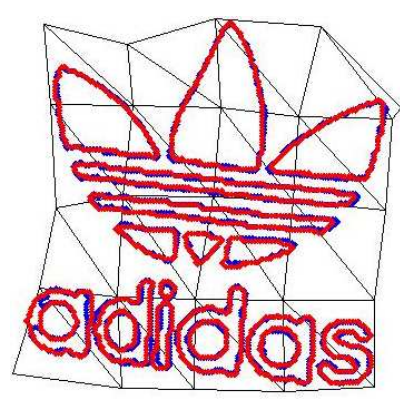

(c)

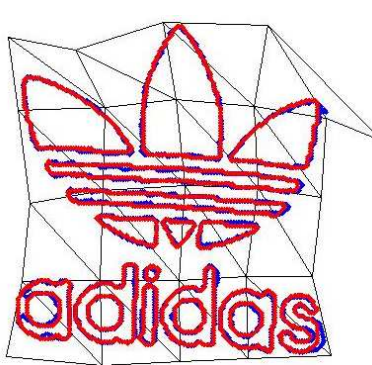

(g)

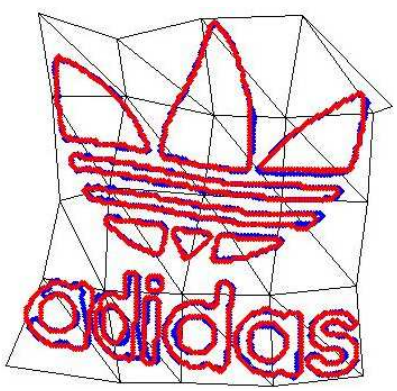

(d)

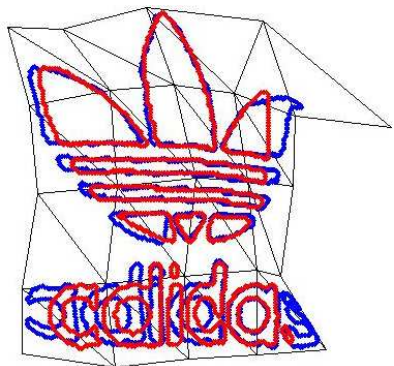

(h)

Figure 7: Mesh deformation corresponding to the example frames in Figure 6 estimated using the error function defined in equation (10)

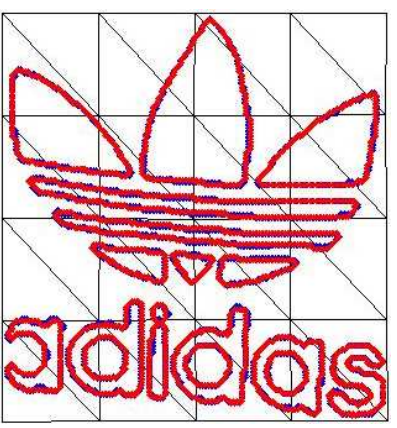

(a)

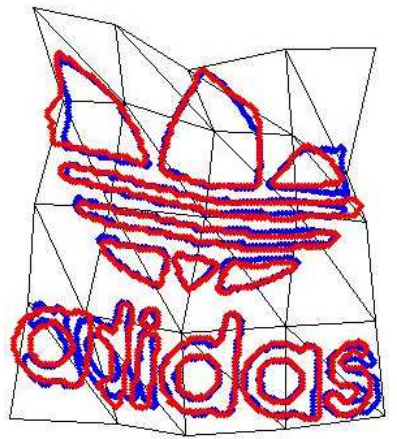

(e)

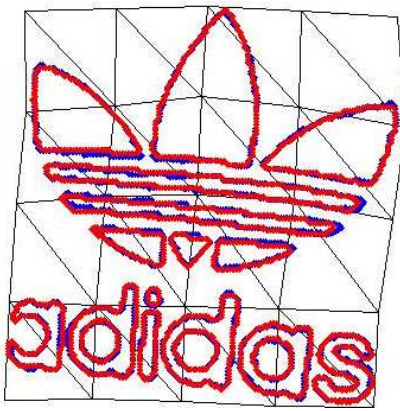

(b)

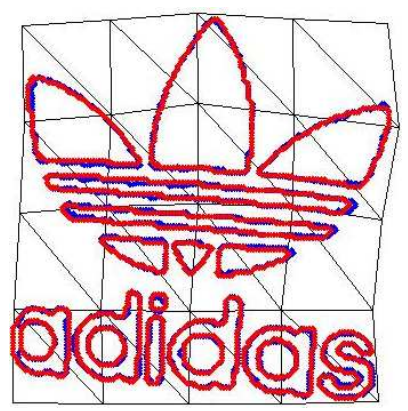

(f)

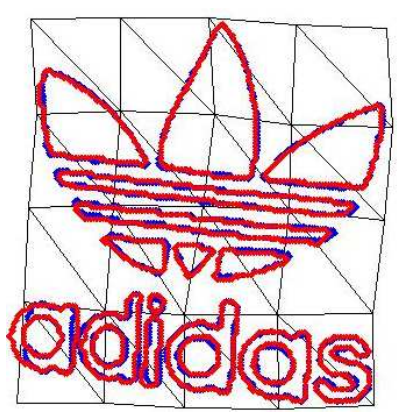

(c)

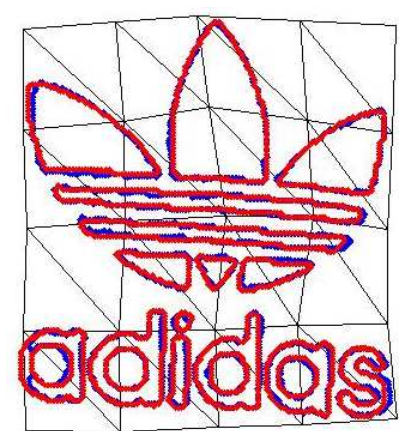

(g)

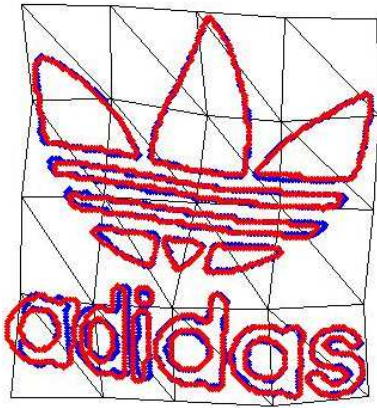

(d)

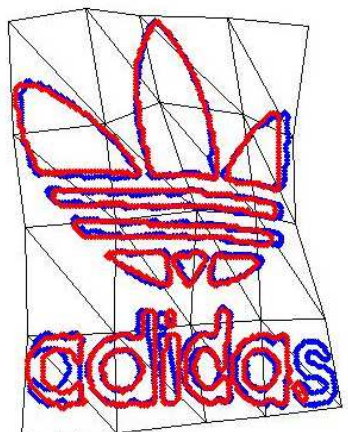

(h)

Figure 8: Mesh deformation corresponding to the example frames in Figure 6 estimated using the error function with additional smoothing constraints defined in equation (11) 